\title{
Релаксация электронного спина и резонансное охлаждение ядерных спинов в структурах GaAs: Mn
}

\author{
(C) А.Е. Евдокимов ${ }^{1}$, М.С. Кузнецова ${ }^{1}$, А.В. Михайлов ${ }^{1}$, К.В. Кавокин ${ }^{1}$, Р.И. Джиоев ${ }^{2}$ \\ ${ }^{1}$ Санкт-Петербургский государственный университет, Лаборатория оптики спина, \\ 198504 Санкт-Петербург, Россия \\ ${ }^{2}$ Физико-технический институт им. А.Ф. Иоффре Российской академии наук, \\ 194021 Санкт-Петербург, Россия \\ E-mail: evdokimov.artyom@gmail.com
}

Поступила в Редакцию 12 апреля 2021 г.

В окончательной редакции 19 апреля 2021 г.

Принята к публикации 19 апреля 2021 г.

\begin{abstract}
Экспериментально изучено поведение времени релаксации спина электрона в объемных слоях GaAs, легированного Mn. Исследовалось изменение степени циркулярной поляризации фотолюминесценции в поперечном и продольном магнитных полях. Обнаружено увеличение времени релаксации электронного спина от 25 нс при слабой накачке до 400 нс при пороговой мощности накачки. Также продемонстрирован эффект резонансного охлаждения ядерной спиновой системы оптически ориентированными электронными спинами.
\end{abstract}

Ключевые слова: структуры GaAs:Mn, поперечное и продольное магнитные поля, спиновая релаксация электронов.

DOI: 10.21883/FTP.2021.09.51287.14

\section{1. Введение}

Понимание механизмов спиновой релаксации в твердых телах является предпосылкой к развитию спинтроники. В более ранних экспериментах было показано, что время спиновой релаксации электронов в $n$-GaAs в диапазоне концентраций доноров $10^{15}-10^{16} \mathrm{~cm}^{-3}$ составляет порядка или больше 100 нс [1-3]. Такой величины уже достаточно для решения некоторых проблем при обработке квантовой информации. В то же время релаксация электронного спина в $p$-GaAs, как и в узких квантовых ямах, обусловленная механизмом Бира-Аронова-Пикуса (БАП), оказывается существенно более быстрой даже при низких температурах $\left(\tau_{s}=1 \mathrm{Hc}[4,5]\right)$. В работе [6] показано, что легирование $p$-GaAs магнитными акцепторами $\mathrm{Mn}$ позволяет существенно подавить спиновую релаксацию БАП за счет компенсации магнитных моментов локализованных дырок и $d$-электронов Mn. При этом на первый план выходят механизмы спиновой релаксации, характерные для полупроводников $n$-типа проводимости: спин-орбитальное взаимодействие и сверхтонкое взаимодействие со спинами ядер решетки [1].

В настоящей работе подробно исследована спиновая релаксация электронов и их взаимодействие с ядерными спинами в $p$-GaAs, легированном Mn.

\section{2. Методика эксперимента}

Исследуемая структура представляла собой объемный слой $\mathrm{GaAs}$, легированного Mn, выращенный методом жидкофазной эпитаксии на подложке GaAs c ориента- цией (001). Степень легирования образца составляет $N_{\mathrm{Mn}}=3 \cdot 10^{17} \mathrm{~cm}^{-3}, N_{d}-N_{a}=3.5 \cdot 10^{15} \mathrm{~cm}^{-3}$, где $N_{\mathrm{Mn}}$, $N_{d}$ и $N_{a}$ - концентрации акцепторов $\mathrm{Mn}$, мелких доноров и акцепторов соответственно. Образец помещался в криостат замкнутого цикла и охлаждался до температуры жидкого гелия. Для создания поперечного или продольного магнитных полей (геометрия Фарадея и Фойгта соответственно) использовался электромагнит, установленный снаружи криостата.

Экспериментально исследовались электронная спиновая динамика с помощью измерения степени поляризации фотолюминесценции (ФЛ) в поперечном магнитном поле (эффект Ханле) и восстановление спиновой поляризации электронов в продольном магнитном поле (polarization recovery curve, PRC).

Фотолюминесценция возбуждалась перестраиваемым непрерывным Ti:Sapphire-лазером с энергией фотона $E_{\mathrm{exc}}=1.55$ эВ. Лазерный пучок фокусировался на образце в пятно диаметром $\sim 200$ мкм. Для модуляции поляризации оптической накачки использовался электрооптический модулятор, который позволяет осуществлять быстрое переключение между правой $\left(\sigma^{+}\right)$и левой $\left(\sigma^{-}\right)$ циркулярными поляризациями света. Сигнал ФЛ разлагался в спектр с помощью двойного монохроматора и регистрировался кремниевым фотодиодом.

Интенсивности компонент поляризации $\sigma^{+}$и $\sigma^{-}$детектировались с помощью двухканального счетчика фотонов. Степень циркулярной поляризации определялась как $\rho_{c}=\left(I^{++}-I^{-+}\right) /\left(I^{++}+I^{-+}\right)$, где интенсивности $I^{++}\left(I^{-+}\right)$определяются как $\sigma^{+}$-поляризованная ФЛ при $\sigma^{+}\left(\sigma^{-}\right)$-поляризованном возбуждении.

На рис. 1 представлены характерные спектры ФЛ для образца GaAs:Mn, измеренные в зависимости от мощ- 


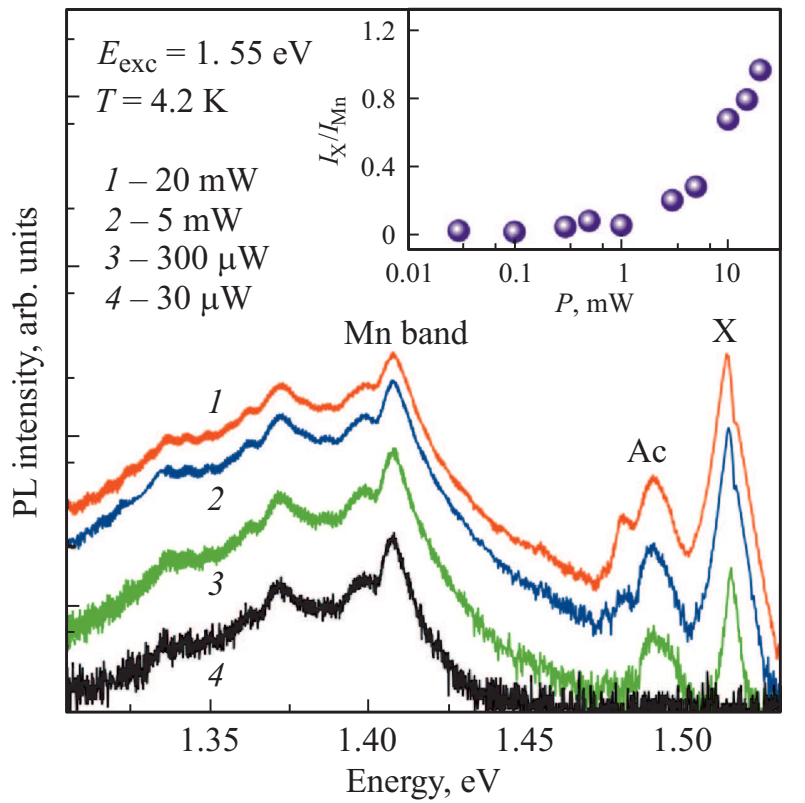

Рис. 1. Спектры фотолюминесценции (PL) при различных мощностях оптического возбуждения $P$. На вставке - отношение пиковых интенсивностей экситонной линии $\mathrm{X}\left(\mathrm{I}_{\mathrm{X}}\right)$ и полосы $\mathrm{Mn}\left(I_{\mathrm{Mn}}\right)$ в зависимости от мощности возбуждения.

ности оптической накачки $P$. Пик фотолюминесценции с энергией 1.51 эВ, обозначенный $\mathrm{X}$, представляет собой излучение свободных и связанных на донорах экситонов. Пик с энергией 1.49 эВ, обозначенный Ас, является результатом переходов зона проводимости-акцептор и мелкий донор-акцептор. При более низких энергиях наблюдается полоса, связанная с переходами на уровни $\mathrm{Mn}$, с максимумом при энергии 1.41 эВ, которая значительно уширена за счет связи с фононами. Эту часть спектров можно интерпретировать как переходы из зоны проводимости и с мелких доноров на акцепторные уровни $\mathrm{Mn}$ и их фононные реплики. Видно, что полоса $\mathrm{Mn}$ наблюдается при всех мощностях накачки, реализуемых в эксперименте, в то время как существует пороговое значение мощности возбуждения в диапазоне $P_{\text {th }}=1-3 \mathrm{мB}$ для появления линий $\mathrm{X}$ и Ac в спектрах ФЛ. Это ярко видно на вставке к рис. 1 , где показано отношение $I_{\mathrm{X}} / I_{\mathrm{Mn}}$ интенсивностей ФЛ для экситонной линии $\mathrm{X}$ и полосы Mn. Спектры ФЛ измерены при достаточно больших мощностях накачки, $10-20 \mathrm{MBT}$, но, как видно из рис. 1 , сдвига экситонной линии не наблюдается, что говорит об отсутствии нагрева образца.

\section{3. Экспериментальные результаты}

\section{1. Кривые деполяризации и восстановления поляризации при модуляции поляризации}

Исследование поведения релаксации электронных спинов на переходах, связанных с марганцем, при мо- дулированном по поляризации оптическом возбуждении позволяет изучать электронные процессы без взаимодействия с ядерной спиновой системой, поскольку при достаточно высоких частотах модуляции, значительно превосходящих обратное время ядерной спиновой релаксации $T_{2}^{-1}\left(T_{2} \sim 10^{-4} \mathrm{c}\right)$ [7], не происходит формирования динамической ядерной поляризации.

Для определения времени релаксации электронного спина $\tau_{s}$ мы измерили серии кривых деполяризации и PRC с частотой модуляции поляризации оптического возбуждения $f_{\text {mod }}=50$ кГц при различных мощностях накачки. Поскольку спины дырок релаксируют намного быстрее, поляризация фотолюминесценции $\rho_{c}$ отражает среднюю спиновую поляризацию электронов. Кривые деполяризации можно описать следующим образом $[4,7]$ :

$$
\rho_{c}=\frac{\rho_{c 0}}{1+\tau / \tau_{s}} \frac{1}{1+\left(B_{x} / B_{1 / 2}\right)^{2}},
$$

где $\rho_{c 0}-$ начальная электронная поляризация, $B_{1 / 2}-$ полуширина на полувысоте (HWHM) кривой деполяризации $B_{1 / 2}=\hbar /\left(g_{e} \mu_{\mathrm{B}} T_{s}\right), \hbar-$ постоянная Планка, $\mu_{\mathrm{B}}$ - магнетон Бора, $g_{e}$ - электронный $g$-фактор (для GaAs $\left.g_{e}=-0.44\right), T_{s}^{-1}=\tau^{-1}+\tau_{s}^{-1}$ - обратное время жизни спина электрона. На рис. 2, $a$ показана серия кривых деполяризации, измеренных при фиксированной частоте модуляции поляризации оптического возбуждения $f_{\text {mod }}=50$ кГц в зависимости от мощности накачки.
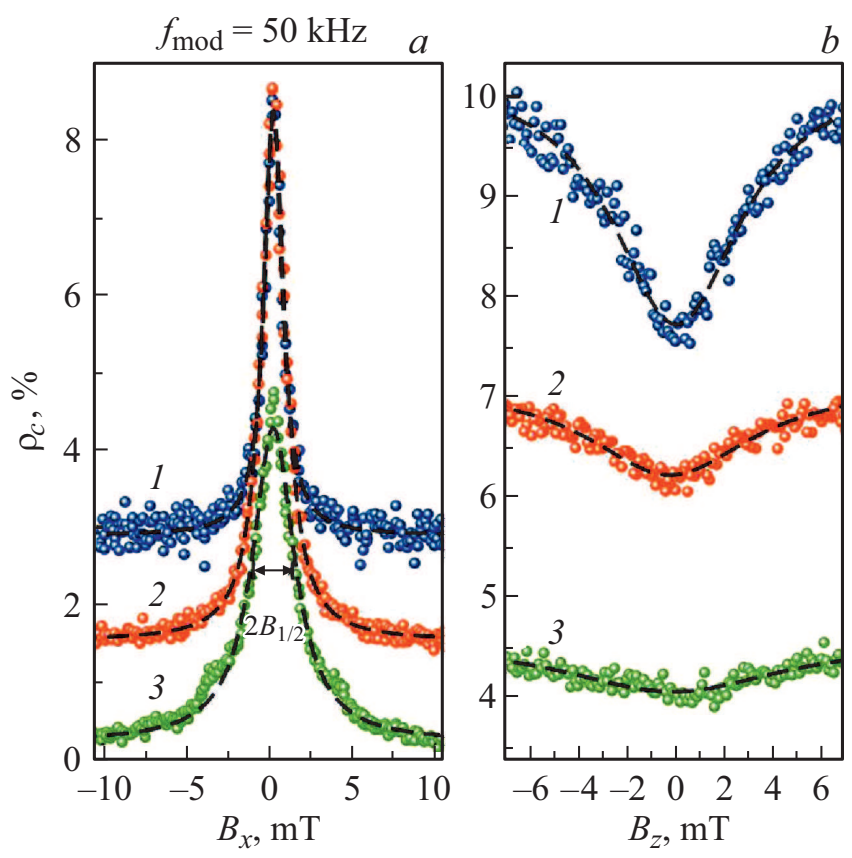

Рис. 2. Кривые деполяризации $(a)$ и кривые PRC $(b)$, измеренные при частоте модуляции $f_{\bmod }=50$ кГц для мощностей накачки 0.5 (1), 5 (2), 15 мВт (3). Кривые деполяризации смещены по вертикали для удобства восприятия. Штриховые линии - аппроксимация экспериментальных кривых по формулам (1) и (2). 


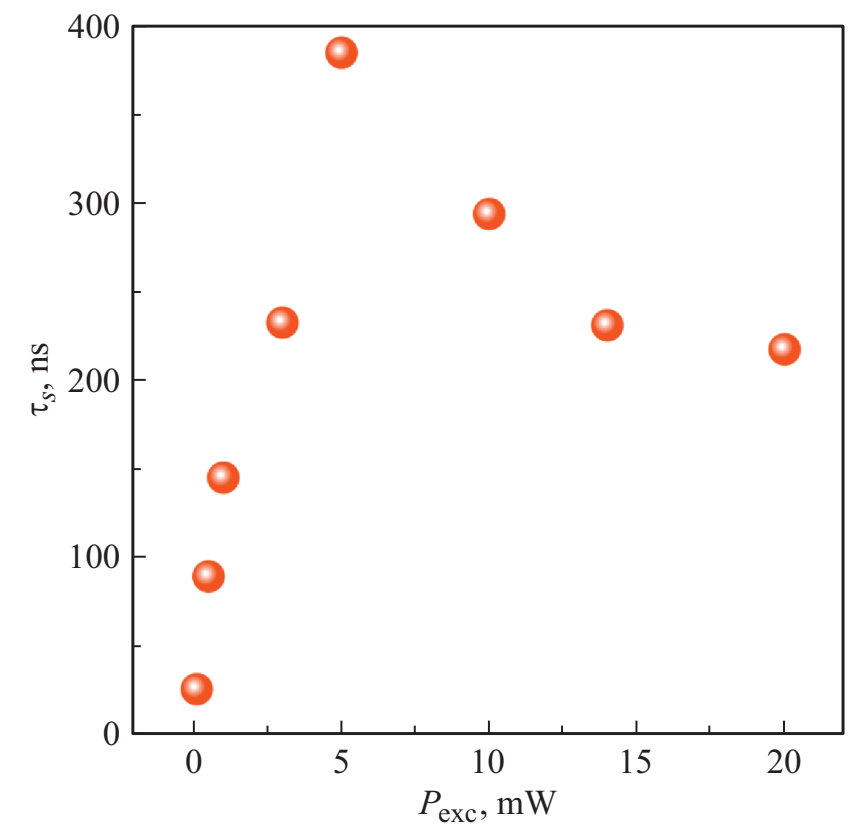

Рис. 3. Зависимость времени релаксации электронного спина от мощности накачки оптического возбуждения.

Весь набор кривых деполяризации, представленных на рис. 2, a, можно с хорошей точностью описать лоренцианами. Параметр $\rho_{c 0}$ был получен из анализа кривых PRC по формуле [6]:

$$
\rho_{c}=\frac{\rho_{c 0}}{1+\tau / \tau_{s}^{*}}, \quad \tau_{s}^{*}=\tau_{s}\left[1+\left(\frac{B}{B_{c}}\right)^{2}\right],
$$

где корреляционное поле $B_{c}=\hbar /\left[\left(g_{A}-g_{e}\right) \mu_{\mathrm{B}}\right] \tau_{c}^{-1}$ и $g_{A}$ зависят от конфигурации акцепторов Мn. На рис. 2, $b$ представлены кривые PRC, измеренные с частотой модуляции $f_{\text {mod }}=50$ кГц в зависимости от мощности накачки. Из анализа экспериментальных данных мы получили мощностную зависимость $\tau_{s}$.

На рис. 3 представлена зависимость $\tau_{s}$ от мощности накачки. Зависимость времени электронной спиновой релаксации от накачки немонотонна. При малых мощностях накачки заряженные акцепторы Mn захватывают фотогенерированные дырки, что приводит к компенсации создаваемого акцепторами флуктуирующего поля, вызывающего спиновую релаксацию. Это приводит к увеличению времени релаксации электронного спина при мощностях накачки ниже $P_{\text {th }}$. При мощности накачки $P_{\text {exc }}=P_{\text {th }}$ все примеси Мn переходят в нейтральную конфигурацию, а $\tau_{s}$ имеет максимальное значение.

При больших мощностях возбуждения все акцепторы $\mathrm{Mn}$ становятся нейтральными, и наблюдается быстрая релаксация спина электрона за счет механизма БАП. Время релаксации электронного спина очень большое для такого типа структур и достигает 400 нс при пороговой мощности накачки.

\section{2. Резонансное охлаждение ядерной спиновой системы}

Как видно из рис. 2, $a$, при большой мощности накачки на кривой деполяризации появляются дополнительные особенности, которые не получается описать одним лоренцианом. На рис. 4 представлена кривая деполяризации, измеренная при частоте модуляции поляризации оптического возбуждения $f_{\text {mod }}=50$ кЦц и мощности оптической накачки $P=15$ мВт. В работе [8] авторы наблюдали аналогичные проявления в спектрах резонансного спинового усиления. Появление таких дополнительных максимумов на кривой деполяризации связаны с резонансным охлаждением ядерной спиновой системы за счет взаимодействия с оптически ориентированными электронными спинами [7]. Магнитные поля, в которых наблюдаются максимумы на кривой деполяризации в наших экспериментах, соответствуют резонансному охлаждению ядер ${ }^{71} \mathrm{Ga}$ и ${ }^{75} \mathrm{As}$.

Рассчитанные значения резонансных магнитных полей для особенностей на кривой деполяризации, экспериментально наблюдаемые при частоте модуляции оптического возбуждения $f_{\text {mod }}=50$ кГц для изотопов ${ }^{69} \mathrm{Ga},{ }^{71} \mathrm{Ga}$ и ${ }^{75} \mathrm{As}$, представлены в таблице.

Как видно из рис. 4, положения центров дополнительных максимумов хорошо соотносятся с расчетными значениями магнитных полей.

Также мы провели серию дополнительных измерений, чтобы проанализировать, как поведение кривой деполяризации изменяется при больших частотах модуляции поляризации оптического возбуждения и изменении температуры. Были измерены серии кривых деполяризации при $f_{\bmod }=200$ кГц при различных мощностях накачки

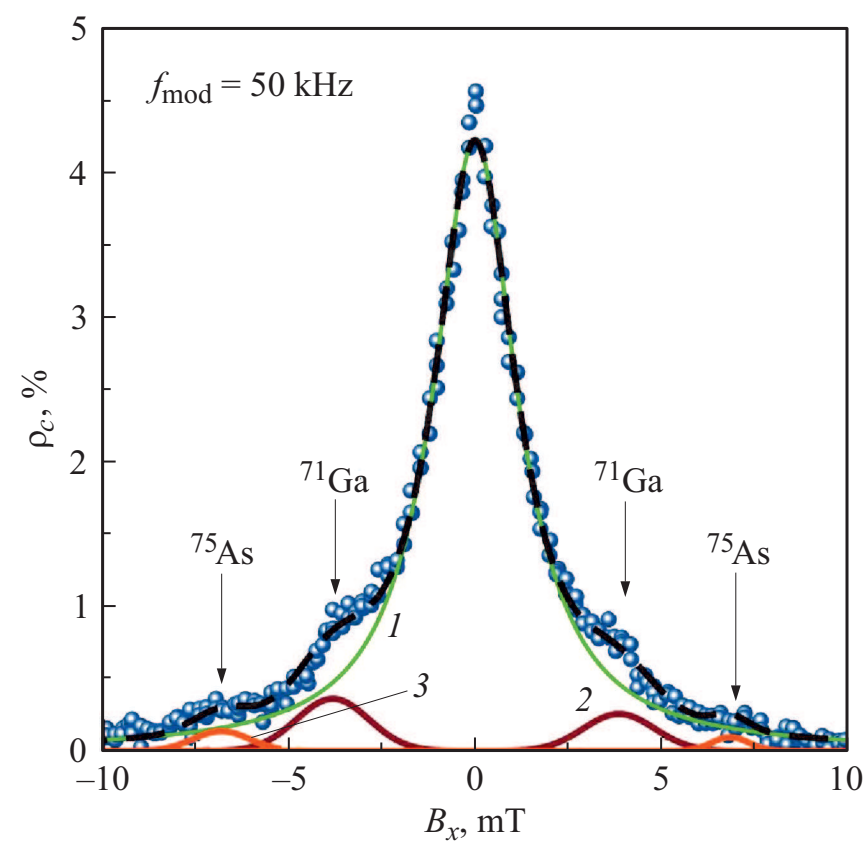

Рис. 4. Кривая деполяризации (точки), электронный пик (1), гауссианы $(2,3)$ соответствуют ядрам ${ }^{71} \mathrm{Ga}$ и ${ }^{75} \mathrm{As}$. 


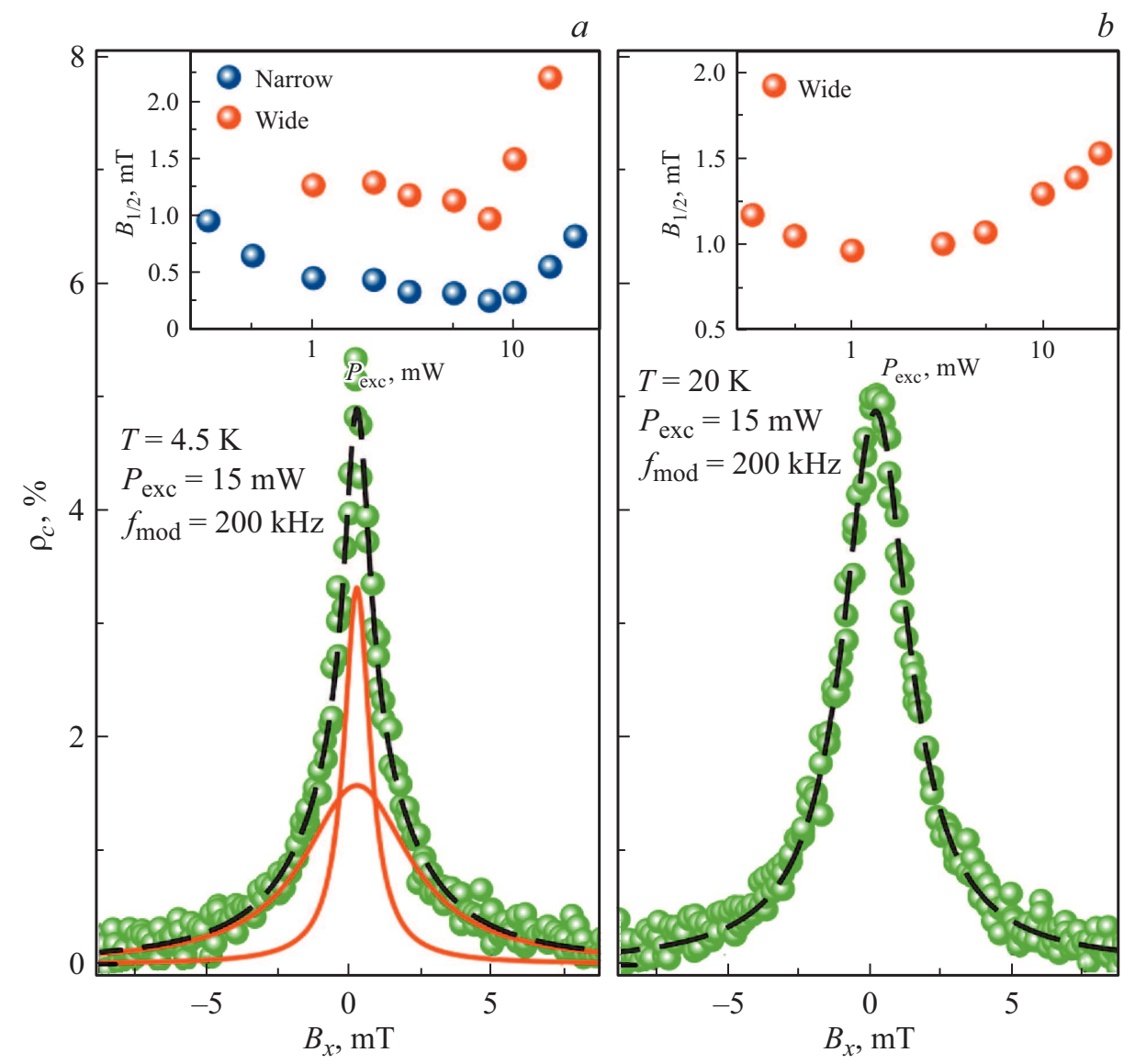

Рис. 5. Кривые деполяризации, измеренные на частоте $f_{\bmod }=200$ кГц и при температурах $4.5(a), 20 \mathrm{~K}(b)$. На вставках зависимости полуширин кривых, входящих в суммарную подгонку кривой деполяризации.

и двух температурах $T=4.5$ и $20 \mathrm{~K}$ (см. рис. 5, $a$ и $b)$. Кривые деполяризации для исследуемой структуры представляют собой довольно узкие линии, полуширины составляют от 0.5 до 2 мТл в зависимости от мощности накачки. При частоте модуляции $f_{\text {mod }}=200$ кГц магнитные поля, при которых должны были бы проявиться особенности, связанные с резонансным охлаждением ядер, выходят за пределы экспериментально измеряемой кривой деполяризации.

На рис. 5, $a$ представлена кривая деполяризации, измеренная при достаточно большой мощности накачки, $P_{\text {exc }}=15$ мВт. Видно, что в суммарную подгонку кривой на рис. 5, $a$ входят два лоренциана. На вставке к рис. 5, $a$ приведена зависимость полуширин кривых деполяризации, измеренных при различных мощностях накачки.

Рассчитанные значения резонансных магнитных полей для изотопов ${ }^{69} \mathrm{Ga},{ }^{71} \mathrm{Ga}$ и ${ }^{75} \mathrm{As}$ при частоте модуляции оптического возбуждения $f_{\text {mod }}=50$ кГц

\begin{tabular}{l|c|c|c}
\hline \multicolumn{1}{c|}{ Изотоп } & ${ }^{71} \mathrm{Ga}$ & ${ }^{69} \mathrm{Ga}$ & ${ }^{75} \mathrm{As}$ \\
\hline $\begin{array}{l}\text { Резонансное } \\
\text { магнитное поле, мТл }\end{array}$ & 3.84 & 4.88 & 6.83
\end{tabular}

При слабых накачках кривая аппроксимируется одним лоренцианом, после порогового значения мощности накачки 1 мВт появляется второй контур.

На рис. 5, $b$ представлена кривая деполяризации, измеренная при $T=20 \mathrm{~K}$. На вставке к рис. 5, $b$ приведена зависимость полуширин кривых деполяризации при различных мощностях накачки. Во всем диапазоне мощностей кривые деполяризации аппроксимируются одним лоренцианом. Это может быть результатом того, что в процессах релаксации участвуют два типа электронов: локализованные и свободные. При увеличении температуры электроны делокализуются, подтверждением является тот факт, что экспериментальные кривые описываются более широким лоренцианом.

\section{4. Заключение}

Времена релаксации электронного спина в GaAsструктурах с акцепторами Мn ведут себя более сложным образом, чем в структурах $p$-типа, и по порядку величин могут быть сопоставимыми со временами релаксации в структурах $n$-типа. В процессах релаксации участвуют два типа электронов, свободные и локализованные, вклад одного из которых пропадает с увеличением 
температуры. Кроме того, в этих материалах ярко проявляется эффект резонансного охлаждения ядерных спинов, что делает их перспективными для исследования электронно-ядерной спиновой динамики.

\section{Финансирование работы}

Работа выполнена при финансовой поддержке DFG в рамках Международного центра совместных исследований TRR 160 (проект № A6) и Российского фонда фундаментальных исследований (грант № 19-52-12043). MCK благодарит Санкт -Петербургский государственный университет за финансовую поддержку в рамках гранта № ID 73031758. ABM благодарит Российский фонд фундаментальных исследований (грант № 19-52-12059).

\section{Конфликт интересов}

Авторы заявляют, что у них нет конфликта интересов.

\section{Список литературы}

[1] R.I. Dzhioev, K.V. Kavokin, V.L. Korenev, M.V. Lazarev, B.Ya. Meltser, M.N. Stepanova, B.P. Zakharchenya, D. Gammon, D.S. Katzer. Phys. Rev. B, 66, 245204 (2002).

[2] V.V. Belykh, K.V. Kavokin, D.R. Yakovlev, M. Bayer. Phys. Rev. B, 96, 241201 (2017).

[3] J.G. Lonnemann, E.P. Rugeramigabo, M. Oestreich, J. Hübner. Phys. Rev. B, 96, 045201 (2017).

[4] G.E. Pikus, A.N. Titkov. Optical Orientation, ed. by F. Meier, B.P. Zakharchenya (Amsterdam, North-Holland, 1984) p. 73.

[5] J. Zhou, M.W. Wu. Phys. Rev. B, 77, 075318 (2008).

[6] G.V. Astakhov, R.I. Dzhioev, K.V. Kavokin, V.L. Korenev, M.V. Lazarev, M.N. Tkachuk, Yu.G. Kusrayev, T. Kiessling, W. Ossau, L.W. Molenkamp. Phys. Rev. Lett., 101, 076602 (2008).

[7] I.A. Merkulov, V.G. Fleisher. Optical Orientation, ed. by F. Meier, B.P. Zakharchenya (Amsterdam, North-Holland, 1984) p. 173.

[8] E.A. Zhukov, A. Greilich, D.R. Yakovlev, K.V. Kavokin, I.A. Yugova, O.A. Yugov, D. Suter, G. Karczewski, T. Wojtowicz, J. Kossut, V.V. Petrov, Yu.K. Dolgikh, A. Pawlis, M. Bayer. Phys. Rev. B, 90, 085311 (2014).

Редактор Л.В. Шаронова

\section{Electron spin relaxation and resonant cooling of nuclear spins in GaAs: Mn structures}

\author{
A.E. Evdokimov' ${ }^{1}$, M.S. Kuznetsova ${ }^{1}$, A.V. Mikhailov' ${ }^{1}$, \\ K.V. Kavokin ${ }^{1}$, R.I. Dzhioev ${ }^{2}$
}

${ }^{1}$ St. Petersburg State University, Spin Optics Laboratory, 198504 St. Petersburg, Russia

${ }^{2}$ Ioffe Institute,

194021 St. Petersburg, Russia

\begin{abstract}
The behavior of the electron spin relaxation time in bulk GaAs layers doped with Mn was studied experimentally. We investigated the dependence of the degree of circular polarization of photoluminescence in transverse and longitudinal magnetic fields. An increase in the relaxation time of the electron spin is found from $25 \mathrm{~ns}$ at low pumping power to $400 \mathrm{~ns}$ at the threshold pumping power. The effect of resonant cooling of the nuclear spin system by optically oriented electron spins is demonstrated.
\end{abstract}

